\title{
ZUR FRÜHGESCHICHTE DES THEMATISCHEN VERZEICHNISSES. LUDWIG RITTER VON KÖCHELS „WISSENSCHAFTLICHE“ SYSTEMATISIERUNG
}

\author{
Thomas HOCHRADNER
}

\begin{abstract}
In the 19th century - starting with Ludwig Ritter von Köchel's Chronologisch-thematisches Verzeichniss sämmtlicher Tonwerke Wolfgang Amade Mozart's - philologically elaborated Thematic Catalogues completed the inventory of music as well as publisher's- and auction catalogues as a third category of specification of a musical repertoire. Köchel's procedure has been considered as pioneer work in the introduction of a fundamental and very specific kind of text. However, the principle of classification for his systematics put at the beginning is - typical for his approach in general - on the one hand based upon handwritten catalogues of Mozart's work and on the other hand influenced by the conventions of the previously edited extended publisher's catalogues of the works of Ludwig van Beethoven and Felix Mendelssohn Bartholdy at Breitkopf \& Härtel's, Leipzig. Thus Köchel's work must be regarded as a strategy following up tendencies of his time that - despite the respect it deserves due to its completeness and accuracy - appears as an almost accidental basis.
\end{abstract}

\section{Zusammenfassung}

Zu Musikinventaren und Verlags- bzw. Auktionskatalogen treten im 19. Jahrhundert, beginnend mit dem Chronologischthematischen Verzeichniss sämmtlicher Tonwerke Wolfgang Amade Mozart's durch Ludwig Ritter von Köchel, philologisch erarbeitete Thematische Werkverzeichnisse als eine dritte Kategorie des musikalischen Repertoireverzeichnisses hinzu. Köchels Vorgangsweise wird als Pionierleistung einer grundlegenden und fachspezifischen Textsorte gesehen. Das Einteilungsprinzip seiner vorangestellten Systematik fußt jedoch - wie grundsätzlich sein Vorgehen - einesteils auf handschriftlichen Werkverzeichnissen zu Mozarts Schaffen, andernteils in Konventionen der kurz zuvor bei Breitkopf \& Härtel in Leipzig aufgelegten, im Vergleich zu früher stark ausgeweiteten Verlagskataloge zu Ludwig van Beethoven und Felix Mendelssohn Bartholdy. So besehen erweist sich Köchels Arbeit als weitergedachte Strategie seiner Zeit, die bei allem Respekt vor seinem Streben nach Vollständigkeit und Exaktheit nicht bewusst, sondern fast zufällig Grundlagen setzte.

Als sich neben Musikinventaren und Verlags- bzw. Auktionskatalogen Mitte des 19. Jahrhunderts mit Thematischen Werkverzeichnissen (= Thematischen Werkkatalogen) eine dritte Form des musikalischen Repertoireverzeichnisses etabliert, wird zugleich eine wissenschaftlich fundierte Verzeichnisstrategie eingeführt. Beschreibung, Typifizierung und Gattungszuordnung musikalischer Werke folgen nunmehr nicht allein Maßgaben der Funktionalität (Musikinventare) und der Vermarktung (Verlagskataloge), sondern dienen auch dem systematisierten Überblick über ein Gesamtœuvre (Thematische Werkverzeichnisse). 
Dabei stellt die Anordnung nach musikalischen Gattungen eine naheliegende Möglichkeit dar, das Verzeichnis der musikalischen Werke eines bestimmten Komponisten in zweckmäßige und überschaubare Rubriken einzuteilen, sofern die Quellenlage nicht eine chronologische Reihung nahelegt und demzufolge die systematische Übersicht lediglich flankierend zum Hauptteil erscheint. Als Prototyp des wissenschaftlichen Thematischen Werkverzeichnisses gilt Ludwig Ritter von Köchels Chronologisch-thematisches Verzeichniss sämmtlicher Tonwerke Wolfgang Amade Mozart's. Nebst Angabe der verloren gegangenen, unvollendeten, übertragenen, zweifelhaften und unterschobenen Compositionen desselben (Leipzig: Breitkopf \& Härtel 1862), wobei Köchel eine einführend vorangestellte, prinzipiell nach Gattungen angeordnete knappe Übersicht mit einem chronologischen Verzeichnis als Hauptteil verband. In allen revidierten Neuauflagen wurde dieses Konzept grundsätzlich beibehalten. Köchels Leistung, die vielfach als Pioniertat gerühmt wird, soll im Folgenden durch einen Vergleich mit zeitgenössischen, von Musikverlagen aufgelegten Werkverzeichnissen neu gewichtet werden. Es wird sich zeigen, dass sich das wissenschaftliche Vorgehen mit aus der Verlagspraxis herausschälte.

Frühe Beispiele für das gedruckte Thematische Verzeichnis bieten die Kataloge des Verlags Breitkopf im 18. Jahrhundert, die in ihrer Anlage dem Inventar noch nahestehen und eine Gruppierung nach Gattungen entweder mit der Funktion oder der Besetzung der Werke verknüpfen. In Inventaren bleibt diese Praxis weiterhin erhalten, sofern sie nicht als „Eingangsbuch“ in der Reihenfolge der Neuanschaffungen geführt werden. Während sich „Funktion“ als Ordnungskriterium der Thematischen Verzeichnisse allmählich verliert, bleibt „Besetzung“ als bedeutsame Kategorie bestehen und bildet bis zum Ende des 19. Jahrhunderts für gewöhnlich die wesentliche Einteilungsgrundlage des sog. „systematischen Verzeichnisses“. Musikalische Gattungen spielen demgegenüber eine untergeordnete Rolle - eine Zurückhaltung, die durchaus widerspiegelt, dass die zeitgenössische deutschsprachige Musikgeschichtsschreibung spärlich mit dem Gattungsbegriff operierte. ${ }^{1}$

Eine vorläufige (noch $\mathrm{zu}$ komplettierende) Bibliographie von Thematischen Werkverzeichnissen ${ }^{2}$ aus dem 19. Jahrhundert hebt zahlenmäßig drei Komponisten heraus: Mozart Mendelssohn - Beethoven.

$\begin{array}{lll}6 & \text { Mozart } & 1805(1828), 1841,[1856], 1862,1876,1889 \\ 5 & \text { Mendelssohn } & {[1852],[\text { ca. 1860], 1868, 1882, 1876 }} \\ 4 & \text { Beethoven } & 1851,1868,1876,1890 \\ 3 & \text { Schubert } & {[1852], 1868,1874} \\ 3 & \text { Liszt } & 1855,1877,1887 \\ 2 & \text { Chopin } & {[1852], 1888} \\ 2 & \text { Schumann } & {[1863], 1868} \\ 2 & \text { Brahms } & 1887,1897 \\ 1 & \text { Moscheles } & {[1861]}\end{array}$

1. Selbst in der ästhetischen Diskussion bleibt der Begriffsgebrauch uneinheitlich. Vgl. Hermann Danuser, Art. Gattung, in: MGG, 2. Auflage, hg. v. Ludwig Finscher, Sachteil Bd. 3, Kassel etc. 1995, Sp. 1042-1069, bes. Sp. 1055.

2. Für bibliographische Hinweise vgl. den Anhang. 


$\begin{array}{lll}1 & \text { Franz } & 1868 \\ 1 & \text { Reinecke } & 1889\end{array}$

Dass Mozart-Werkkataloge hier zu Beginn stehen und an Zahl überwiegen, täuscht über den eigentlichen Sachverhalt hinweg. Es handelt sich 1805 (Neuauflage 1828) um den Druck von Mozarts eigenhändigem Werkverzeichnis bei André, 1841 um dessen Bestandskatalog von MozartAutographen (in Vorbereitung der Veräußerung der Sammlung) und 1856 um Stages Verkaufskatalog (des Anteils von Jean Baptiste André) - mithin um drei Kataloge, die keine Konventionalisierung bewirkten, obwohl sie zusammen mit dem Werkverzeichnis in Nissens Biographie sowie den handschriftlichen Werkverzeichnissen von A. [Anton] André (1833) und Aloys Fuchs (1837, später ergänzt von Joseph Hauer) für Köchel eine wertvolle Hilfestellung (und mitunter eine verführerische Fehlerquelle) darstellten. Ergänzend und darüber hinaus folgte Köchel den Ausführungen bei Otto $\mathrm{Jahn}^{3}$ und wertete zusätzlich die damals im „Dommusikverein und Mozarteum“ in Salzburg verwahrten Mozart-Briefe aus den Nachlässen der Mozart-Söhne aus.

Zuvor bereits hatten Breitkopf \& Härtel mit der Veröffentlichung der im Auftrag des Hauses erstellten Thematischen Verzeichnisse zu Beethoven (1851), Mendelssohn (1852) und Liszt (1855) einen Maßstab für das Komponisten-Werkverzeichnis gesetzt. ${ }^{4}$ Für das Beethoven-Verzeichnis anhand der Korrespondenz mit Breitkopf \& Härtel belegbar, sind Köchel wohl alle drei Verzeichnisse bekannt gewesen. Eben dies dürfte in der Folge bewirkt haben, dass er sich für die Drucklegung seines „MozartCatalogs“5 an Breitkopf \& Härtel wandte, allerdings erst, nachdem das Manuskript vollständig abgeschlossen war. ${ }^{6}$ Köchels durchgehend chronologisches Prinzip - die ihm vorliegenden gedruckten und handschriftlichen Mozart-Werkkataloge (Mozarts eigenhändiges Werkverzeichnis, André 1833, André 1841) führten es nur teilweise durch - orientiert sich vermutlich mittelbar an der Reihung nach Werken mit Opuszahlen bzw. Werken ohne Opuszahlen, welcher die drei genannten BreitkopfVerzeichnisse folgen. Gemessen an deren Standard handelt es sich bei Köchels Versuch des streng chronologischen Prinzips um eine Ersatzlösung, da Opuszahlen zu Mozarts Werken keine Orientierung gaben. Doch gelang es Köchel nicht, das chronologische Prinzip geschlossen anzuwenden. Er musste vielfach zu einem Sternchen als Zeichen der ungefähren Datierung greifen, und erst Alfred Einstein löste 1937 in der von ihm bearbeiteten dritten Auflage des Köchel-Verzeichnisses ${ }^{7}$ die offenen Datierungen auf (mit akribischer Überlegung, doch nicht fehlerfrei, wie wir heute wissen).

Deiters)

3. Otto Jahn, W. A. Mozart, 4 Bände, Leipzig 1856-59, Leipzig ${ }^{2} 1867-69$, Leipzig ${ }^{3} 1889-91$ (bearb. v. Hermann

4. Das Verzeichnis für Chopin 1852 entstand in Koproduktion mit den Verlagen Brandus in Paris und Wessel in London und erhielt eine andere Anlage.

5. So in der Korrespondenz des Verlags Breitkopf \& Härtel in Leipzig mit Ludwig Ritter von Köchel (Depot im Staatsarchiv Leipzig, Bestand „Verlag Breitkopf \& Härtel“), Brief an Köchel vom 9. August 1861, Kopierbuch 131, S. 367.

6. Ein Briefwechsel zwischen Köchel und dem Verlag Breitkopf \& Härtel ist vor 1860 nicht zu belegen. Zwar wird im selben Jahr der „Thematische Catalog“ zu Mozarts Werken erwähnt, doch ohne vorerst eine Drucklegung bei Breitkopf \& Härtel in Aussicht zu nehmen. Darüber wurde offenbar erst 1861 bei einem Besuch Köchels im Leipziger Verlagshaus verhandelt und Einigung erzielt. - Dazu siehe den Brief an Köchel vom 26. April 1861, Kopierbuch 131, S. 180f.

7. Chronologisch-thematisches Verzeichniss sämmtlicher Tonwerke Wolfgang Amade Mozart's. Nebst Angabe der verloren gegangenen, unvollendeten, übertragenen, zweifelhaften und unterschobenen Compositionen desselben, 3. Auflage, bearb. v. Alfred Einstein, Leipzig 1937. 


\begin{tabular}{|c|c|c|c|c|c|c|c|}
\hline Verzeichnis & \begin{tabular}{|l|} 
Incipits \\
(im Hauptteil)
\end{tabular} & \begin{tabular}{|l|} 
Takt- \\
zahlen \\
\end{tabular} & $\mathbf{E Z}$ & \begin{tabular}{|l|} 
Quellen- \\
beschreibung
\end{tabular} & Bemerkungen & Verweise & Preisangaben \\
\hline Mozart (André) 1841 & durchwegs & nein & ja & in Ansätzen & Besetzung & nein & extra \\
\hline Beethoven 1851 & durchwegs & nein & nein & nein & $\begin{array}{l}\text { Besetzung, Widmung, } \\
\text { Bearbeitungen }\end{array}$ & Ausgaben (sporadisch) & ja \\
\hline Chopin [1852] & durchwegs & nein & nein & nein & $\begin{array}{l}\text { Tonart, Widmung, } \\
\text { Bearbeitungen }\end{array}$ & Ausgaben & ja \\
\hline Mendelssohn 1852 & durchwegs & nein & nein & nein & Besetzung, Widmung & Ausgaben & ja \\
\hline Schubert 1852 & durchwegs & nein & nein & nein & Besetzung & nein & ja \\
\hline Mozart (Stage) [1856] & zumeist & nein & zumeist & in Ansätzen & nach Jahn Bd. 1 & André 1841, Jahn Bd. 1 & ja \\
\hline Mendelssohn ca. 1860 & durchwegs & nein & nein & nein & Besetzung & Ausgaben & ja \\
\hline Moscheles 1861 & \begin{tabular}{|l} 
durchwegs \\
\end{tabular} & nein & nein & nein & Besetzung & Ausgaben & ja \\
\hline Köchel 1862 & durchwegs & ja & $j a$ & $\begin{array}{l}\text { in Ansätzen, } \\
\text { aber nur zum } \\
\text { Autograph }\end{array}$ & $\begin{array}{l}\text { Besetzung, Widmung, } \\
\text { Anmerkungen (meist nach } \\
\text { Jahn) }\end{array}$ & $\begin{array}{l}\text { frühere Kataloge, } \\
\text { Ausgaben, } \\
\text { Literatur (bes. Jahn) }\end{array}$ & nein \\
\hline Schumann 1863 & durchwegs & nein & nein & nein & Besetzung & Ausgaben & ja \\
\hline $\begin{array}{l}\text { Beethoven } 1868 \\
\text { (Nottebohm) }\end{array}$ & durchwegs & nein & $\begin{array}{l}\text { in An- } \\
\text { sätzen }\end{array}$ & ja & $\begin{array}{l}\text { Besetzung, } \\
\text { Widmung, Anmerkungen, } \\
\text { Bearbeitungen }\end{array}$ & Erstausgabe, Ausgaben & ja \\
\hline $\begin{array}{l}\text { Schubert, Schumann, } \\
\text { Mendelssohn, Franz } 1868\end{array}$ & nein & nein & nein & nein & Besetzung, Opuszahl & nein & ja (Anhang) \\
\hline $\begin{array}{l}\text { Schubert } 1874 \\
\text { (Nottebohm) }\end{array}$ & durchwegs & nein & $\begin{array}{l}\text { in An- } \\
\text { sätzen }\end{array}$ & nein & $\begin{array}{l}\text { Besetzung, Widmung, } \\
\text { Anmerkungen, Bearbeitungen }\end{array}$ & Erstausgabe, Ausgaben & ja \\
\hline
\end{tabular}


Eine detailliertere Übersicht zu den bis 1874 erschienenen Thematischen Werkverzeichnissen zeigt den außergewöhnlichen Standort des Köchel-Verzeichnisses auf. Es steht fraglos in der Tradition der zuvor verfassten Mozart-Werkkataloge (insbesondere von André 1833 und 1841), doch baute Köchel den Apparat an zusätzlichen Informationen aus. Sein „Mozart-Catalog“ steht - ich wage dies als These - im Bann des Mythos Mozart, dem er zugleich entgegenwirken wollte. Denn festzustellen, welche Fassung die ,authentische“ war und wo dagegen eine Bearbeitung vorlag sowie gesicherte Kompositionen von zweifelhaften und unterschobenen zu scheiden, war neben dem versuchten chronologischen Nachvollzug des Schaffens der hauptsächliche Antrieb seines Unternehmens. Betrachtet man den Informationsgehalt des einzelnen Eintrags, wird diese Absicht daran deutlich, dass Köchel im Unterschied zu den Breitkopf-Werkverzeichnissen die Taktzahlen der Werke bzw. Sätze vermerkt. Hinzu kommen ferner Quellenbeschreibungen, die freilich nicht kritisch erschlossen sind, sondern auszugsweise Notizen aus den Originalhandschriften wiedergeben, und Anmerkungen, die im Großen und Ganzen auf eine Wiederholung der Erkenntnisse von Jahn hinauslaufen. Hinter der Widmung des Köchel-Verzeichnisses an Jahn verbirgt sich demnach mehr als kollegiale Dankbarkeit oder ehrfürchtige Anerkennung: nämlich das Eingeständnis einer umfassenden Abhängigkeit.

Was in der Tabelle nach heutigem Verständnis mit „Bearbeitungen“ angeführt ist, nämlich die Umarbeitung eines Werkes für eine andere Besetzung, erscheint in zeitgenössischer Terminologie als „Übertragung“ und wird damit von einer „Bearbeitung“ abgesetzt, mit der die kompositorische Faktur verändert wird. Sowohl „Übertragungen“ als auch „Bearbeitungen“ schloss Köchel aus seinen Nachweisen aus. Dennoch griff er Tendenzen der Breitkopf-Werkverzeichnisse auf, nicht nur, was die optische Präsentation des einzelnen Eintrags anbelangt. Summarisch führen bereits die BreitkopfWerkverzeichnisse Literatur im Anhang auf, neben Angaben zu Portraits, Büsten und Widmungsträgern. Schließlich nennt Köchel zwar im Gegensatz zu den Breitkopf-Werkverzeichnissen keine Preisangaben - doch hatte er diese zunächst vorgesehen und erst bei einem abschließenden Korrekturgang durchwegs gestrichen. ${ }^{8}$

Köchels Einteilung der vorgeschalteten „Uebersicht der vollständigen Compositionen nach Gattung und Zahl“ (S. 1) umfasst 23 Werkgruppen (sie werden für die spätere [Alte] Mozart-Ausgabe übernommen; Laufzeit der Drucklegung 1877-1905). Darin erscheint sein Gattungsbegriff an ältere Rubrizierungen gebunden: er umfasst - mehrfach zuerst genannte - musikalische Gattungen in Verbindung mit Besetzungsvorgaben. Überraschend schimmert die barocke Stiltrias einer Musik für Kirche, Bühne und Kammer durch, die Köchel aber nicht aus überlieferten Musikinventaren gewonnen haben kann, da er für seine Vorstudien kein einziges solches Musikinventar benützte. Vielmehr dürfte eine Orientierung an Raphael Georg Kiesewetters 1834 veröffentlichter Musikgeschichte den Ausschlag für eine musikhistorisch überlegte Annäherung gegeben haben. ${ }^{9}$ Angeleitet wurde Köchel aber in Sonderheit vom Ideal des vollständigen Werkes, dessen ursprüngliche Gestalt er nachzuweisen trachtete - ob es in diversen Inventaren aufschien, war daher unerheblich. Dass sich aus nicht

8. Ergebnis einer Sichtung von Köchels Druckvorlage (Wien, Archiv der Gesellschaft der Musikfreunde, 2742 b / 46).

9. Siehe dazu Thomas Hochradner, Ludwig Ritter von Köchel und das Konzept des Werkverzeichnisses, in: Musikkonzepte - Konzepte der Musikwissenschaft. Bericht über den Internationalen Kongreß der Gesellschaft für Musikforschung Halle (Saale) 1998, hg. v. Kathrin Eberl und Wolfgang Ruf, Band II: Freie Referate, Kassel u. a. 2000, S. 9-15. 
erhaltenen Quellen z. B. die Verbreitung eines Werkes besser nachvollziehen lässt und so wertvolle Einsichten zur Überlieferungsgeschichte greifbar werden, blieb ebenso außen vor wie das Bestreben, einen Überblick über das gesamte Werk eines Komponisten zu gewinnen. In Köchels „MozartCatalog“ erscheinen „Verloren gegangene Compositionen“ und „Unvollständige“ bloß in einem Anhang, im zehn Jahre später publizierten Werkverzeichnis für Johann Joseph Fux ${ }^{10}$ wird auch darauf verzichtet. Köchels Werkverzeichnisse zu Mozart und Fux boten daher im wesentlichen ,nur“ eine Übersicht zu vollständigen Werken. Für Mozart gliedert Köchel in:

I. Messen, Requiem

II. Litaneien, Vespern

III. Offertorien, Kyrie, Te Deum, Veni, Regina Coeli

IV. Orgelsonaten

V. Cantaten mit Orchesterbegleitung

VI. Opern, theatralische Serenaden u. dergl.

VII. Arien, Trio, Quartette, Chöre mit Orchesterbegleitung

VIII. Lieder mit Klavierbegleitung

IX. Canone für 2-12 Singstimmen

X. Clavier-Sonaten mit Phantasien

XI. Clavier-Variationen

XII. Clavier-Einzelstücke, Minuette, Rondo, Allegro u. dergl.

XIII. Clavier zu 4 Händen und 2 Claviere

XIV. Clavier-Violin-Sonaten, -Variationen

XV. Clavier-Trio, -Quartette, -Quintette

XVI. Streich-Duo und -Trio

XVII. Streich-Quartette und mit 1 [einem] Blasinstrumente

XVIII. Streich-Quintette

XIX. Symphonien

XX. Divertimenti, Serenaden, Cassationen

XXI. Orchesterstücke: Märsche, einzelne Symphoniesätze

XXII. Tänze für Orchester

XXIII. Concerte

Somit ordnete er geistliche Musik vor weltliche und ging innerhalb dieser Ebenen nach Besetzungen vor: bei allen geistlichen Werken und bei weltlichen Vokalwerken tendenziell von größerer zu kleinerer Besetzung, bei Instrumentalwerken aber umgekehrt. Sobald Orchesterbesetzung erreicht ist, tritt eine eigenwillige interne Gewichtung nach Gattungen ein: Symphonien, Divertimenti

10. Ludwig Ritter von Köchel, Johann Josef Fux. Hofcompositor und Hofkapellmeister der Kaiser Leopold I., Josef I. und Karl VI. von 1698 bis 1740, Wien: Alfred Hölder (Beck'sche Universitäts-Buchhandlung) 1872, Nachdruck Hildesheim - New York: Olms 1974, ${ }^{2}$ 1988, Beilage X: Thematisches Verzeichniss der Compositionen von Johann Josef Fux. 
/ Serenaden / Cassationen, Märsche / Einzelsätze, Tänze, Concerte. Verglichen mit dem Bestandsverzeichnis von André 1841, das lt. „Vorbericht“ „in Classen abgetheilt“ ist, zeigen sich Unterschiede:

A. Kirchenmusik. / Oratorien, Messen, Vespern, Lytanien etc.

B. Opern und Theater-Musik. / (Dahin gehören auch einzelne Opern-Arien, Duetten, Terzetten und Quartetten.)

C. Concert-Arien mit Orchester-Begleitung.

D. Lieder und Solfeggien mit Clavier-Begleitung.

E. Sinfonieen und Ouvertüren für Orchester.

F. Divertimenti, Serenaden, Märsche für Streich- u. Blasinstrumente.

G. Harmoniemusik.

H. Violinmusik. / Concerte, Quartette und Quintette etc. Divertimenti und Fugen.

I. Claviermusik. / Concerte, Quartette und Terzette, Sonaten mit Violin, Solo-Sonaten etc.

K. Musik für Orgel.

L. Musik für Flöte.

M. Musik für Oboe.

N. Musik für Horn.

O. Musik für Harfe.

P. Musik für Harmonica.

Q. Tanzmusik.

R. Authentische Abschriften Mozart'scher Sinfonieen in Stimmen, welche Mozart behufs der Aufführung mit auf Reisen nahm und deren Correctur er selbst besorgte, so wie hie und da die Tempi und Vortragszeichen anmerkte oder einzelne Orchester-Stimmen eigenhändig schrieb.

Besetzungskriterien sind in diesem 17 Gruppen umfassenden Verzeichnis deutlich bestimmender als später bei Köchel. Je instrumentenspezifisch werden Gattungen, z. B. „Concerte, Quartette und Quintette etc.“ der „Violinmusik“, rubriziert. Köchel, im Grunde ein genialer Kompilator, machte sich dieses Muster nur bedingt zu Nutze und fächerte die Einteilung stärker nach Gattungen auf." Sein Vorgehen unterscheidet sich aber auch von den wenige Jahre vor dem Erscheinen des „Mozart-Catalogs“ publizierten Breitkopf-Werkkatalogen. Diese Veröffentlichungen boten ihm zwar mancherlei Anregung, doch übernahm er deren Anlage nicht generell, obwohl das „Systematisch geordnete Verzeichniss“ - das im Beethoven-Verzeichnis 1851 als Anhang erscheint und analog zur Präsentation der Werke ohne Opuszahl im Sinne eines Findbuches gestaltet ist - für die weiteren bei Breitkopf \& Härtel aufgelegten Verzeichnisse und auch darüber hinaus maßgeblich wurde. Die Einteilung lautet hier: nicht klären.

11. Ob er sich dazu ein anderes ihm vorliegendes hs. Verzeichnis zum Vorbild genommen hat, lässt sich gegenwärtig 
Instrumentalmusik.

A. Für das Orchester.

B. Für die Violine mit Orchester, Septette, Sextette, Quintette, Quartette, Trios.

C. Für Blasinstrumente.

D. Für das Pianoforte mit und ohne Begleitung.

1. Für das Pianoforte mit Orchester, Quintette, Quartette, Trios, Duos.

2. Für das Pianoforte zu 4 Händen.

3. Für das Pianoforte allein.

Gesangmusik.

1. Messen, Oratorien, Opern, Cantaten und andere Gesangstücke mit Begleitung des Orchesters oder mehrerer Instrumente.

2. Lieder und Gesänge mit und ohne Begleitung des Pianoforte.

Instrumentalmusik erhält gegenüber Vokalmusik den Vorrang. Im Weiteren läuft die Systematik auch hier durchwegs von größerer zu kleinerer Besetzung, die „Gesangmusik“ ist alphabetisch nach Titeln, bei Liedern und Gesängen nach Titeln und Textanfängen überkreuzt verzeichnet. In ähnlicher Weise gibt das Mendelssohn-Verzeichnis 1852 Gattungen als untergeordnete Kategorie von Besetzungen wieder. 
ron

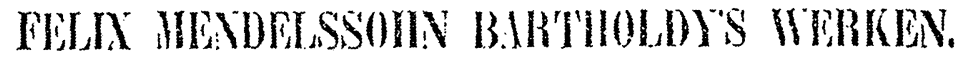

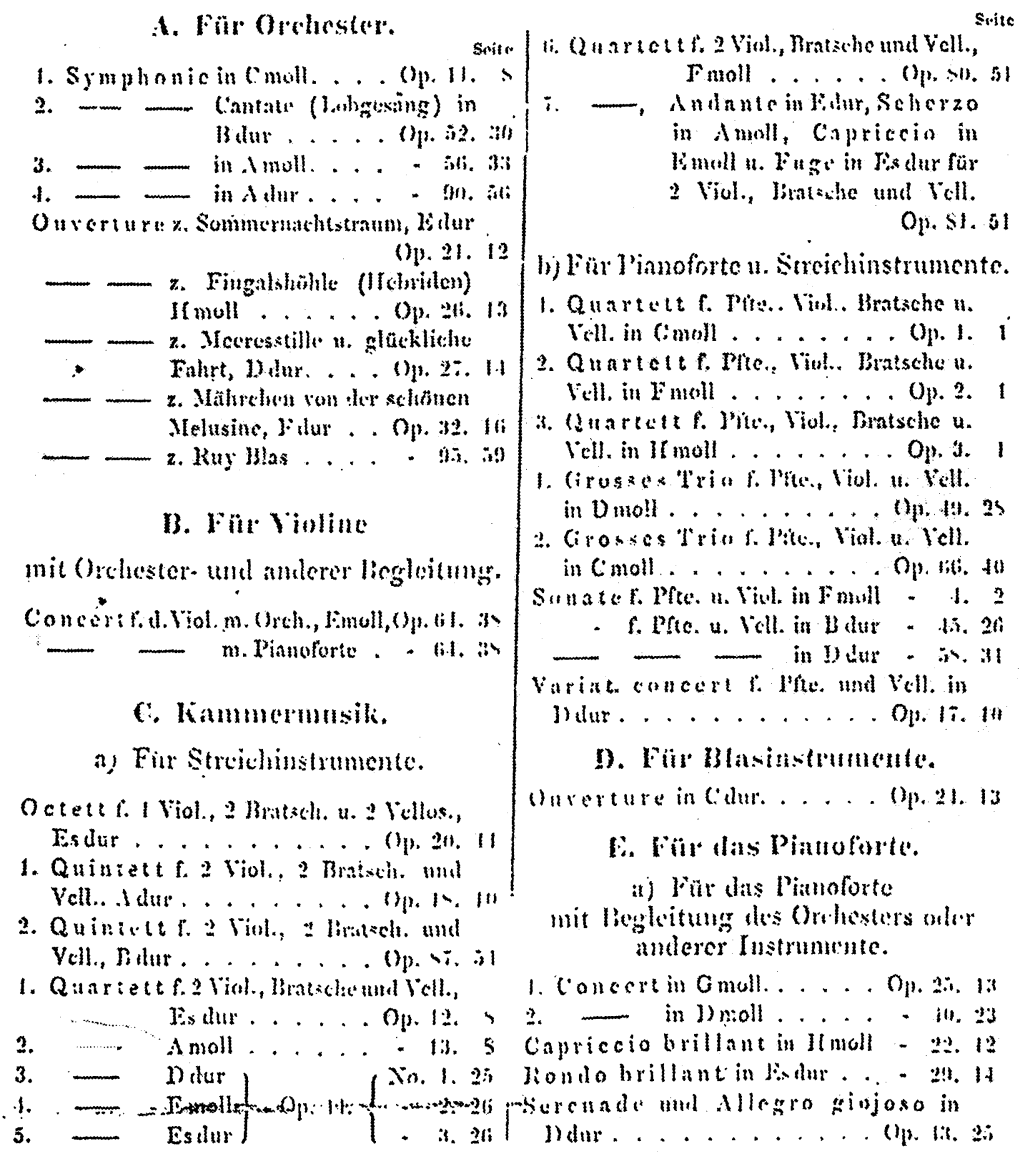

Fig. 2.

Thematisches Verzeichniss im Druck erschienener Compositionen von Felix Mendelssohn Bartholdy, Leipzig: Breitkopf \& Härtel, unwesentlich erweiterte Neuauflage von ca. 1860,

Druckplattennummer 6925, S. 73. 
Dieses trennt jedoch nicht in Instrumental- und Gesangmusik - die Ebene ist ausgelassen -, während zusätzlich der Begriff „Kammermusik“ eingeführt wird. Nicht einzelne Gattungen, sondern Gattungsbündel finden als Oberbegriffe Verwendung. Sie werden entweder explizit genannt oder stillschweigend vorausgesetzt. Hinzu kommt ein gelegentliches Durchbrechen der prinzipiell alphabetischen Abfolge innerhalb der Rubriken: die Symphonie wird der Ouverture vorangestellt (Abschnitt A.). Der von Breitkopf \& Härtel zur Überarbeitung des Beethoven-Verzeichnisses engagierte Gustav Nottebohm hebt dann 1868 Symphonie, Concert und Sonate in den jeweiligen Rubriken vom Rest der genannten Werke ab, wogegen er für sein Schubert-Verzeichnis 1874 streng alphabetisch vorgeht. Außerdem ergänzt er darin das ,Systematisch geordnete Verzeichnis“ mit einem alphabetischen „Register der Instrumentalcompositionen“, einer objektivierten Variante der Präsentation. 
B.

Register der Instrumentalcompositionen.

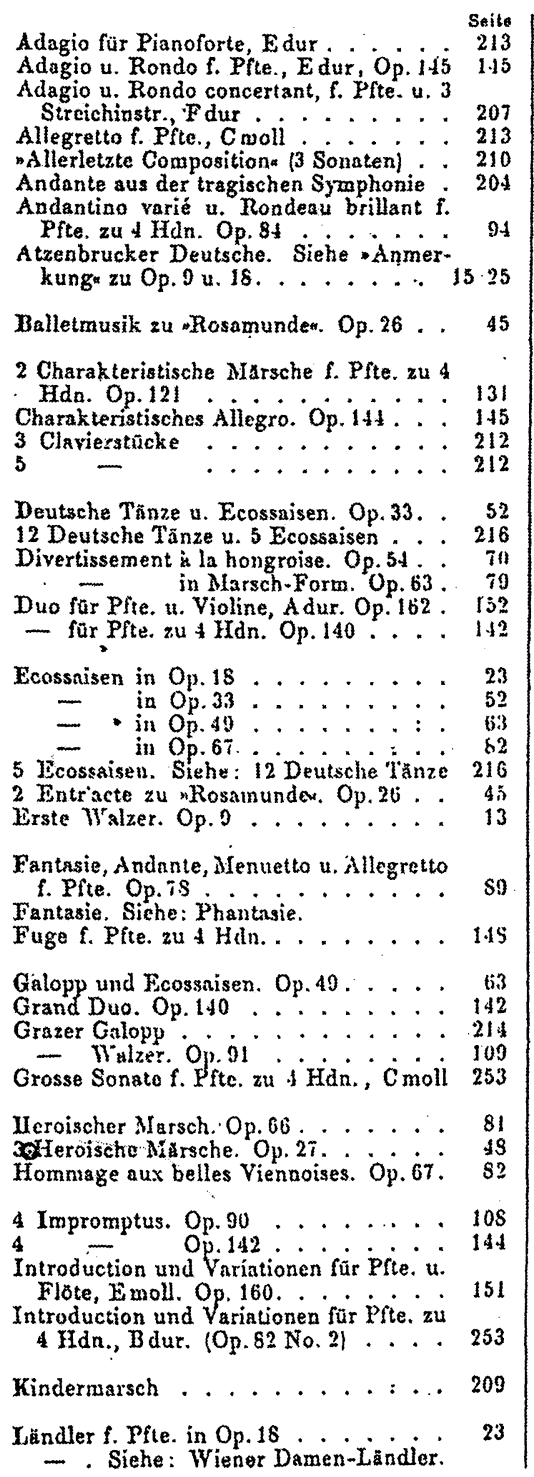

12 Ländler fo pite 12 Iändler f. Pfte. Op. 171 f. Pfte. zu 2 a 4 Hin: : 157 I.ebensstürme. Charakteristisches Allegro f. Pfte zu 4 Hdn. Op. 144. . . . . 145 Letzte Walzer. Op.127...... 135

Marche funèbre, $\mathrm{C}$ moll. Op. $55 \ldots \ldots$ i

héroique, A moll. Op. $66 . \ldots$ si

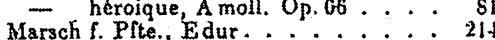
2 Marsche (marches caractéristiques) für 2 Marsche (marches caractéristiques) für
Pfte. zu 4 Hdn. Op. 121 131 3 - $\quad$ Pfte. zu 4 Harches héroiques). Op.27. 4 $3=$ (marches militaires). Op.51: 65 Moments musicals. Op.94 ...... III

Nocturne f. Pfte. Viol. u. Vell. Op. 14S. 140 Notre amitié est invariable. Rondóf. Pfta.

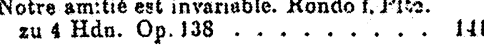

Octett. Op.166......... 154 Originaltanze. Op.y $\ldots \ldots \ldots 13$ Ouverture f. Pfte, zu + Hdn. Op. 34 . . 53 - f. Pfte zu 4 Hdn. Ddur. 200 - im italienischen Styl. Op ini is - zu uAlfonso u. Estrellaw. $O_{p} .6^{3}$ si = zu wFiernbrasm. Op.76. . 5 = $\quad$ zu nRiernbrasn. Op. $16.26 \ldots$

Phantasie f. Pfte u. Violine. Op.159 , 131 - f. Pfte zut Han., Fmoll. Op. 103 - P. Pfte, Cdur. Op. $15 \ldots 2$ 4 Polonnisen f. Píte. zu 1 Hdn. Op. $73: 57$ Quartett, A moll. Op. $29 \ldots \ldots$. . 49 一 Es dur. Op. $125 \ldots \ldots . . .133$ = Gdur. Op.161 ...... 151 $=$ Bdur. Op. $16 \mathrm{~S} \ldots \ldots \ldots 156$ $=D_{\text {moll }} \ldots \ldots \ldots 205$ $=$ Gmoll $_{\ldots} \ldots \ldots \ldots 210$ $\mp \quad$ Ddur $\ldots \cdots \cdots$ Quartett-Satz, c moll $\therefore \therefore \ldots$ 2n Quintett, $\mathrm{Cdur.}$ Op: $163 \ldots \ldots$ 152 - f. Yfte. u. Streichinstr.; Adur Op.114......... 127

Reitermarsch. Op. 121 No.1 ... . . 131 Reliquie, unvollendete Sonate $; 211$ Rondeau brillant f. Pfte. u. Viol. Op. 70 St - - f. Pfte. zu 4 Hdn. Op. 84 Rondo f. Pfte. zu 4 Hdn., A dur. Op. $107 \quad 123$ $T$ f. Pfte, zu 4 Hdn., D dur. Op. 138 iH Rondo. Siehe: Adagio u. IRondo. Rosamunde, MIusik dazu. Op. 26 . . . , 45

2 Satze oiner unvollendeten Symphonie in $\mathrm{H}$ moll. . . ........ 2104 2 Scherzi für Pianoforte $\ldots \ldots 213$

Sehnsuchtswalzer. Op. 9 No.2 $\ldots \ldots 13$

Sonate f. Píte. A moli. Op.42 ... 58

Fig. 3.

Thematisches Verzeichniss der im Druck erschienenen Werke von Franz Schubert, hg. v. G. [Gustav] Nottebohm, Wien: Friedrich Schreiber 1874 [S. 288: „Druck von Breitkopf und Härtel in Leipzig.“], S. 270. 
Sie korrespondiert dem alphabetischen Register der Titel und Textanfänge von Vokalwerken, das ohnedies zum Standard solcher Verzeichnisse gehörte. Ein solches „Register der Instrumentalcompositionen“ erscheint indes nur im Schubert-Verzeichnis 1874. Somit zeigt sich, dass es keine wirkliche Alternative zum „Systematischen Verzeichnis“ bot.

Grundsätzlich ist damit der vorgegebene Rahmen abgesteckt, sieht man vom ChopinVerzeichnis 1888 ab. Dessen Novitäten werden im Vorwort angekündigt: „Im Anhang finden sich ausser einer Uebersicht auf Friedrich Chopin bezüglicher Bücher, Schriften, Büsten, Portraits etc. ein systematisches, ein nach Tonarten und ein chronologisch geordnetes Werkverzeichnis mit Angabe der deutschen, französischen und englischen Original-Verleger nebst entsprechenden Preisen und Hinzufügung der Widmungsempfänger, Text- und Titelregister der Gesänge, Liste der Widmungen und drei nach dem von Hans Schmidt veröffentlichten, stufenweise geordneten Verzeichnisse bearbeitete übersichtliche Zusammenstellungen der Werke Chopin's in aufsteigender Schwierigkeit, sowie eine Anzahl Verzeichnisse von Ausgaben verschiedener Verleger." Die hier neu vorgeschlagene Lösung zur Systematisierung nach technischem Anspruch fand meines Wissens keine Nachfolge.

Betrachtet man die genannten Publikationen im Hinblick auf die Verwendung des Gattungsbegriffs, ist

a) die Funktion der Systematik die einer Nachschlagehilfe, die mit musikalischen Gattungen lose operiert, aber im Hintergrund durchaus davon getragen wird - Köchels „Uebersicht“, in der Gattungen durch die Positionierung der Gattungsbegriffe hervorgehoben sind, stellt insofern eine Neuerung dar;

b) eine konsequente Klassifikation nach einzelnen Gattungen findet nicht statt, im Gegenteil wird neben „Organen der Ausführung“ (so heißt es im Schumann-Verzeichnis 1863 für „Besetzung“) nach sonstigen Alternativen gesucht;

c) eine Qualifikation nach Gattungen leuchtet punktuell hervor, wenn Symphonie, Sonate und Konzert aus der alphabetischen Anordnung herausgenommen und abgesondert werden;

d) zur Präzisierung (d. h. näheren Unterscheidung) wird auf der untersten Ebene der Systematik der Werktitel herangezogen, wobei es sich bei Instrumentalwerken meist um Gattungsbegriffe als Stellvertreter eines spezifischen Titels handelt.

Sowohl Paul Graf Waldersee ${ }^{12}$ als auch Alfred Einstein ${ }^{13}$ ließen das systematische Verzeichnis Köchels nahezu unverändert. Einstein entfernte es jedoch von seinem ursprünglichen Platz am Beginn des Verzeichnisses und fügte es dem Anhang ein. In der sechsten Auflage ${ }^{14}$ wurde es wieder an den Anfang gestellt und teils neu formuliert, wobei nun musikalische Gattungsbegriffe konsequenter als in der Erstauflage des Köchelverzeichnisses im Vordergrund stehen, da sie stets zuerst genannt werden. Doch die Koppelung mit Besetzung bleibt erhalten. Geistliche Musik wird vor weltlicher verzeichnet, scheint es zunächst. Auf Vokalmusik folgt Instrumentalmusik, aber die Systematik ist

12. 2. Auflage, bearb. v. Paul Graf von Waldersee, Leipzig 1905.

13. 3. Auflage, bearb. v. Alfred Einstein, Leipzig 1937.

14. Bearb. v. Franz Giegling, Alexander Weinmann und Gerd Sievers, Wiesbaden 1964. 
mehr denn je inkonsistent. Die „Übersicht“ beginnt zwar mit geistlichen Vokalwerken, doch sind später die Kirchensonaten (Gruppe 16) an vorerst uneinsichtiger Stelle angeführt, nicht zu Beginn der Instrumentalwerke. In der Instrumentalmusik geht die Präsentation zu einer Kombination mit Besetzungen über. Von einer Systematik nach musikalischen Gattungen lässt sich folglich nicht sprechen, und folgerichtig fällt das Wort ,systematisch“ auch nicht in der Überschrift. Tatsächlich handelt es sich um die Gliederung der Neuen Mozart-Ausgabe, der aber die Serien-Titel fehlen. Gattungsbündel sind wiederum ausschlaggebend für das Raster, doch im Vergleich zur Erstauflage des Köchelverzeichnisses werden sie diffuser gefasst, weil die maßgeblichen Kriterien der Gesamtausgabe einem eigenen Konzept folgten. Für die Präsentation der erwähnten Kirchensonaten als Werkgruppe 16 nach den Konzerten könnte ihre allmähliche Entwicklung bis hin zum verkappten Orgelkonzert (KV 336/336d) den Ausschlag gegeben haben.

Thematische Übersicht der Kompositionen W. A. Mozarts

Serie I: Geistliche Gesangswerke

1) Messen und Requiem

2) Litaneien, Vespern

3) Kleinere Kirchenwerke

4) Oratorien, geistliche Singspiele und Kantaten

Serie II: Bühnenwerke

5) Opern und Singspiele

6) Musik zu Schauspielen, Pantomimen und Balletten

7) Szenen, Arien und Chöre mit Orchester

Serie III: Lieder, mehrstimmige Gesänge und Kanons

8) Lieder mit Klavier, Mandoline oder Orgel

9) Mehrstimmige Gesänge

10) Kanons

Serie IV: Orchesterwerke

11) Sinfonien

12) Cassationen, Serenaden, Divertimenti und Einzelstücke für Streich- und Blasinstrumente

13) Märsche für Orchester / Tänze für Orchester (oder für Klavier)

Serie V: Konzerte

14) Konzerte für ein oder mehrere Soloinstrumente

15) Konzerte für ein oder mehrere Klaviere

Serie VI: Kirchensonaten

16) Kirchensonaten 
Serie VII: Ensemblemusik für größere Solo-Besetzungen

17) Divertimenti, Serenaden und Einzelstücke für Blasinstrumente allein

18) Divertimenti und Einzelstücke für Streichinstrumente allein

Serie VIII: Kammermusik

19) Streich-Quintette und Quintette mit Blasinstrumenten

20) Streich-Quartette und Quartette mit Blasinstrumenten

21) Trio und Duo für Streich- und Blasinstrumente

22) Quintette, Quartette und Trio mit Klavier und mit Glasharmonika

23) Sonaten und Variationen für Klavier und Violine (oder Flöte, oder Violoncello)

Serie IX: Klaviermusik

24) Werke für zwei Klaviere und für Klavier zu vier Händen

25) Sonaten, Fantasien und Rondi für Klavier

26) Variationen für Klavier

27) Einzelstücke für Klavier, Glasharmonika oder Orgelwalze

Serie X: Supplement

Diversa

$[\ldots]$

Ein Werkverzeichnis richtet sich nach der Zielgruppe aus. Wie sich die BreitkopfWerkkataloge vor allem an den Musikhandel und weiter an die musikalische Praxis wandten, so die wissenschaftlich-kritischen Werkverzeichnisse nach dem Zweiten Weltkrieg an die Musikforschung. Ein verallgemeinerndes Resumé verbietet sich demnach. Je mehr sich das Musikleben zum Konzertwesen hin verlagerte und Informationen über erhältliche Musikalien verzichtbar machte, desto stärker konnte sich der wissenschaftliche Ansatz durchsetzen. Andere Werkverzeichnisse gehen dabei weit konsequenter nach musikalischen Gattungen vor als das Köchelverzeichnis in seinem Vorspann. Die Verbindung zur Gesamtausgabe aber liegt für ein Werkverzeichnis nahe und der Gedanke ist nicht von der Hand zu weisen, dass Köchel schon bei Vorbereitung seines Verzeichnisses an eine nachfolgende Mozart-Gesamtausgabe dachte, für deren Zustandekommen er vierzehn Jahre später beträchtliche Eigenmittel zur Verfügung stellen sollte. ${ }^{15}$ Die Breitkopf-Werkkataloge unternehmen dagegen in Hinsicht auf die wenig später von Breitkopf \& Härtel initiierten Serienausgaben von Beethoven und Mendelssohn keine vergleichbare Sichtung des jeweiligen Werkbestands (die Beethoven-Gesamtausgabe wird 1862-65, die Mendelssohn-Ausgabe erst 1874-77 bei Breitkopf \& Härtel publiziert).

15. Siehe dazu bes. Otto Biba, Ludwig Ritter von Köchel (1800-1877). Zur Erinnerung an seinen vor 100 Jahren erfolgten Tod, in: Österreichische Musikzeitschrift 32 (1977), Heft 7/8, S. 310-317; ders., Ludwig Ritter von Köchels Verdienste um die Mozart-Gesamtausgabe, in: Bürgerliche Musikkultur im 19. Jahrhundert in Salzburg, hg. v. Rudolph Angermüller, Salzburg 1981, S. 93-103; Wolfgang Rehm, Nochmals: Ritter von Köchels Verdienste um die „Alte Mozart-Ausgabe“, in: Neue Musik und Tradition. Festschrift Rudolf Stephan zum 65. Geburtstag, hg. v. Josef Kuckertz u. a., Laaber 1990, S. 171-178. 
Indem Köchel mit seiner Distanzierung von den Breitkopf-Werkkatalogen eine spezifische Entwicklung einleitete, wird verständlich, warum Einstein ihm nach einer Kritik in Sachfragen, die durchaus schärfer hätte ausfallen können, attestiert: „Aber sein Fleiß, seine Begeisterung, sein Idealismus haben ihn doch weit über die bloße Zusammenfassung der Arbeit seiner Vorgänger hinausgeführt.“ Eine gewisse - für seine Zeit erstaunliche - Weitsicht im Gattungsdenken ist dem hinzu zu fügen. Doch legt ein Vergleich der Thematischen Werkverzeichnisse aus dem 19. Jahrhundert klar:

a) dringlicher als eine Unterscheidung nach Gattungen wird allgemein die Feststellung der authentischen und vollständigen Werke und daran anknüpfend bei Köchel die Erfassung der Werke in ihrer Originalgestalt erachtet;

b) das Einteilungsprinzip bildet sich durch eine Kombination von Besetzung und musikalischer Gattung und erscheint in verschiedenen Schattierungen;

c) die unterschwellige Bedeutung musikalischer Gattungen als Sammelbegriff ist nicht zu unterschätzen;

d) Verlage und Musikalienhandlungen versuchen, die Interessen des Klientels zu bündeln, indem sie sich mit einzelnen Komponisten gewidmeten Werkverzeichnissen, die besonders auf den Verkauf der im Verlag erschienenen Musikalien abgestimmt sind, die beginnende wissenschaftliche Auseinandersetzung geschäftlich zu Nutze machen;

e) die handschriftliche Abfassung privater Inventare bleibt von den Neuerungen in der Verzeichnisstrategie nahezu unberührt - nach wie vor bildet hier meist die Funktion eines Werkes das entscheidende Kriterium der Einteilung.

Jede Katalogisierung des Musikrepertoires repräsentiert auch das „musikalische Gedächtnis“, weil sie den Nachweis von bzw. Zugang zu Beständen erst gewährleistet. Im Laufe des 19. Jahrhunderts fächert sich ein bis dahin gemeinsames Verfahren auf, da nun Musikpraxis, Musikalienhandel und Musikwissenschaft je eine spezifische Handhabe zur Erfassung des Werkbestands suchen. Doch wurde Europa damals nicht nur - durch den internationalen Musikalienhandel - von einem unifizierenden Repertoire überzogen. Zugleich brachte das sprunghaft steigende Musiksammlertum mit sich, dass etliche Quellen ihren Besitzer wechselten und eine Vielzahl neuer Abschriften älterer Werke entstand. Mit dieser Entwicklung vermochten die gedruckten Komponisten-Werkverzeichnisse damals - gleichgültig, ob aus Verkaufsinteresse oder aus wissenschaftlichem Antrieb vorgelegt - nicht Schritt zu halten. 


\section{Im Text erwähnte gedruckte Werkverzeichnisse in chronologischer Reihenfolge ihres Erscheinens}

Thematisches Verzeichniss sämmtlicher Kompositionen von W. A. Mozart, so wie er solches vom $9^{\text {ten }}$ Februar 1784 an, bis zum $15^{\text {ten }}$ November 1791 eigenhändig niedergeschrieben hat. Nach dem Original-Manuscripte herausgegeben von A. [Anton] André, Offenbach: André 1805.

Neuauflage ebenda 1828.

$63 \mathrm{p}$.

Thematisches Verzeichniss derjenigen Originalhandschriften von W. A. Mozart, [...] welche Hofrath André in Offenbach a. M. besitzt, Offenbach: André 1841.

$77 \mathrm{p}$.

Thematisches Verzeichniss sämmtlicher im Druck erschienenen Werke von Ludwig van Beethoven, Leipzig: Breitkopf \& Härtel 1851.

$167 \mathrm{p}$.

Thematisches Verzeichniss der im Druck erschienenen Compositionen von Friedrich Chopin, Leipzig: Breitkopf \& Härtel / Paris: Brandus / London: Wessel o. J. [1852].

Druckplattennummer 8497, 44 p.

Thematisches Verzeichniss im Druck erschienener Compositionen von Felix Mendelssohn Bartholdy,

Leipzig: Breitkopf \& Härtel [1852].

Druckplattennummer 6925, 84 p.

Thematisches Verzeichniss im Druck erschienener Compositionen von Franz Schubert, Wien: Diabelli [1852].

Druckplattennummer 8932, 49 p.

Reprint unter dem Titel: Anton Diabellis Werkkatalog Franz Schubert (1852), Wien: Krenn 1987 (Wiener Archivstudien 10).

Thematisches Verzeichniss werthvoller meist noch ungedruckter Original-Handschriften W. A. Mozart's, Berlin: Franz Stage [1856].

Ohne Druckplattennummer, $15 \mathrm{p}$.

Thematisches Verzeichniss im Druck erschienener Compositionen von Felix Mendelssohn Bartholdy, Leipzig: Breitkopf \& Härtel [um 1860].

Druckplattennummer 6925, 87 p.

Thematisches Verzeichniss im Druck erschienener Compositionen von Ignaz Moscheles, Leipzig: Kistner [1861].

Druckplattennummer 2531, $66 \mathrm{p}$. 
Zur Frühgeschichte des Thematischen Verzeichnisses. Ludwig Ritter von KöChels „wisSEnSChaftliche“ Systematisierung

Thematisches Verzeichniss sämmtlicher im Druck erschienenen Werke Robert Schumann's mit Inbegriff aller Arrangements etc., Leipzig - (Hamburg) - New York: J. Schuberth \& Co. [1863].

Druckplattennummer 2536, 110 p.

Thematisches Verzeichniss der im Druck erschienenen Werke von Ludwig van Beethoven. Zusammengestellt und mit chronologisch-bibliographischen Anmerkungen versehen von Gustav Nottebohm, Leipzig: Breitkopf \& Härtel 1868.

$220 \mathrm{p}$.

Reprint unter dem Titel Ludwig van Beethoven. Thematisches Verzeichnis von Gustav Nottebohm nebst der Biblioteca Beethoveniana von Emerich Kastner ergänzt von Theodor Frimmel, Leipzig: Breitkopf \& Härtel 1925, S. 1-220.

Verzeichniss sämmtlicher Lieder u. Gesänge von Franz Schubert, Rob. Schumann, Fel. Mendelssohn und Robert Franz. Nach den Titeln und Anfangsworten der Texte alphabetisch geordnet. Anhang: Preisverzeichniss und Uebersicht der Bearbeitungen älterer Gesangwerke von Robert Franz, Halle: Heinrich Karmrodt 1868.

$34 \mathrm{p}$.

Thematisches Verzeichniss der im Druck erschienenen Werke von Franz Schubert, hg. v. G. [Gustav] Nottebohm, Wien: Friedrich Schreiber 1874 [S. 288: „Druck von Breitkopf und Härtel in Leipzig."].

$288 \mathrm{p}$.

Verzeichniss sämmtlicher Werke von W. A. Mozart, L. van Beethoven, F. Mendelssohn Bartholdy, Leipzig 1876 (Breitkopf \& Härtel's Gesammtausgaben musikalischer Classiker). $20 \mathrm{p}$.

Thematisches Verzeichniss der im Druck erschienenen Compositionen von Felix Mendelssohn Bartholdy. Dritte vervollständigte Ausgabe, Leipzig: Breitkopf \& Härtel 1882.

$100 \mathrm{p}$.

Reprint Walluf-Nendeln 1976.

Thematisches Verzeichniss der bisher im Druck erschienenen Werke von Johannes Brahms. Nebst systematischem Verzeichnis und Registern, Berlin: Simrock 1887.

$134 \mathrm{p}$.

Thematisches Verzeichniss der im Druck erschienenen Compositionen von Friedrich Chopin. [...] Neue umgearbeitete und vervollständigte Ausgabe, Leipzig: Breitkopf \& Härtel 1888.

$86 \mathrm{p}$.

Nachtrag zum chronologisch-thematischen Verzeichniss sämmtlicher Tonwerke Wolfgang Amade Mozart's, Leipzig: Breitkopf \& Härtel 1889.

$32 \mathrm{p}$.

Anuario Musical, 59 (2004)

189

(c) Consejo Superior de Investigaciones Científicas

Licencia Creative Commons 3.0 España (by-nc)

http://anuariomusical.revistas.csic.es 
Verzeichniss der bis jetzt im Druck erschienenen Compositionen von Carl Reinecke. Zusammengestellt und herausgegeben von Franz Reinecke, im Selbstverlag des Herausgebers 1889 [Bezug: „Leipzig: Breitkopf \& Härtel“].

$76 \mathrm{p}$.

Verzeichniss der Musikalischen Autographe von Ludwig van Beethoven sowie einer Anzahl von alten, grossentheils vom Meister mit eigenhändigen Zusätzen versehenen Abschriften im Besitze von A. [August] Artaria in Wien. Auf Grundlage einer Aufnahme Gustav Nottebohm's neuerlich durchgesehen von Prof. Dr. Guido Adler, Wien: Artaria 1890.

$22 \mathrm{p}$.

Thematisches Verzeichniss sämmtlicher im Druck erschienenen Werke von Johannes Brahms. Nebst Systematischem Verzeichniss und Registern, Berlin: N. Simrock 1897.

$175 \mathrm{p}$.

Reprint einer angeblich vierten Auflage von 1907 [nicht feststellbar, entpuppt sich als Konvolut der späteren Auflagen des Verzeichnisses mit weiteren Ergänzungen], hg. v. Joseph Braunstein, New York 1956; weiterer Reprint unter dem Titel: The N. Simrock Thematic Catalog of the Works of Johannes Brahms [Thematisches Verzeichniss sämmtlicher im Druck erschienenen Werke von Johannes Brahms]. New Introduction, including Addenda and Corrigenda, by Donald M. McCorkle, New York 1973. 